\title{
Two- and Three-dimensional Mid-Infrared Chemical Imaging
}

\author{
Fabio Zobi ${ }^{\star a}$ and Martin Obst ${ }^{b}$
}

\begin{abstract}
This article describes the state-of-the-art in 2D and 3D tomographic mid-IR imaging and its current and potential applications in the imaging of material and biological systems with a focus on cells and tissues. 2D FTIR microscopy is first presented in terms of the basic experimental configurations of the technique, optics, data and image acquisition. This first section provides a basis for the ensuing discussion on 3D FTIR spectromicrotomography. In the second part, examples of 2D and 3D chemical imaging are given with a focus on the use of molecular chemical probes and probe-free applications, respectively.
\end{abstract}

Keywords: Chemical probes · FTIR spectro-microtomography · FTIR 2D and 3D spectroscopy · IR imaging • IR spectromicroscopy · Tomography

\section{Introduction}

Measurements of absorption spectra in the mid infrared (mid-IR) spectral region $\left(\sim 400 \mathrm{~cm}^{-1}-4000 \mathrm{~cm}^{-1}\right)$ are routinely used to characterize the molecular properties of soft matter. Overall, mid-IR absorption spectroscopy is a useful technique for both compositional analysis and the characterization of molecular properties. For the past twenty years, the dominant technology for the measurement of midIR absorption spectra has been Fourier Transform Infrared (FTIR) spectroscopy. The fast measurement times and high signal-to-noise ratios achieved with FTIR spectroscopy have facilitated the development of mid-IR absorption spectroscopy in a microscopy configuration, by allowing the rapid measurement of small sample regions, in the range of $100 \mu \mathrm{m}$ or less. As detailed in the following section, mid-IR microscopy allows for the collection of mid-IR absorption spectra pixel by pixel over an extended 2D area of the sample. The resulting datasets, called spectral hypercubes, can be analyzed to extract the

${ }^{\star}$ Correspondence: Prof. Dr. F. Zobi ${ }^{\mathrm{a}}$

E-mail: fabio.zobi@unifr.ch

aDepartment of Chemistry, University of Fribourg

Chemin du Musée 9, CH-1700 Fribourg

${ }^{b} B a y C E E R$, University of Bayreuth

Dr-Hans-Frisch-Str. 1-3, 95448 Bayreuth, Germany distribution in space of specific absorption bands, providing 2D images based on the spectroscopic properties of the sample. It is common to plot the distribution of absorption bands that are characteristic for some molecules of interest, thus providing images of molecular distribution in the sample. Such plots are often called chemical maps or chemical images of the sample because they represent the variation of its chemical composition in 2D. Since most molecular species give rise to strong and often specific absorption bands in the mid-IR region, IR microscopy can be applied to a wide variety of samples, both in the solid and in the liquid phase. The main limitation for transmission measurements is given by the thickness of the sample, which needs to be of the order of $1 \mu \mathrm{m}$ to $100 \mu \mathrm{m}$ to prevent saturation of the absorption bands.

Recent developments in the field of mid-IR imaging have seen a move towards the implementation of experiments for 3D imaging. Two main approaches are being developed. In one approach, proposed by Petibois and coworkers, an extended sample is sectioned into slices, a few micrometers thick, which are individually imaged.[1] The IR images of all the sections are then assembled into a 3D image of the whole sample using a computer. This approach allows the measurement at high spatial resolution of extended samples, such as a whole mouse brain, and is limited only by the computational power required to handle the datasets, typically of the size of $1 \mathrm{M}$ pixels. However, it can be applied only to samples that are amenable to sectioning.

An alternative approach, proposed by Martin et al., involves the measurement of tilt angle series of transmission images of the same sample, followed by reconstruction of the tomographic image. ${ }^{[2]}$ The approach is limited by the size of the individual sample, which should be thin enough to avoid saturation of mid-IR absorption. However, it can in principle be applied to samples that cannot be sectioned, such as soft or live samples, or to samples which need to be preserved. This article will focus on this latter approach. Furthermore, we will discuss the state-of-the-art in 2D and 3D tomographic mid-IR imaging and its current and potential applications in the imaging of biological systems such as cells and tissue. For 2D FTIR chemical imaging the discussion will be focused on the use of molecular chemical probes that have bridged the gap between live cell IR imaging and inorganic and organometallic chemistry, while mainly probe free applications will be presented as examples of 3D FTIR spectro-microtomography.

\section{IR Spectromicroscopy and IR Imaging}

In a microscopy setup, millimolar (mM) concentrations of chromophores can be detected, but the exact value depends on the oscillator strength of the vibration (i.e. the value of the extinction coefficient) of the specific absorption bands being analyzed. The IR microscope is similar in structure to a conventional optical microscope (Fig. 1) and it can be considered as housing an interferometer and objectives that act as beam condensers. The objectives are used to focus both the IR beam needed for the spectroscopic measurement and the visible light beam used for sample inspection. The optical paths travelled by the two beams are parcentric and parfocal ensuring, therefore, consistent matching of the IR beam location on the sample image and allowing selective measurement of exact spatial locations. 


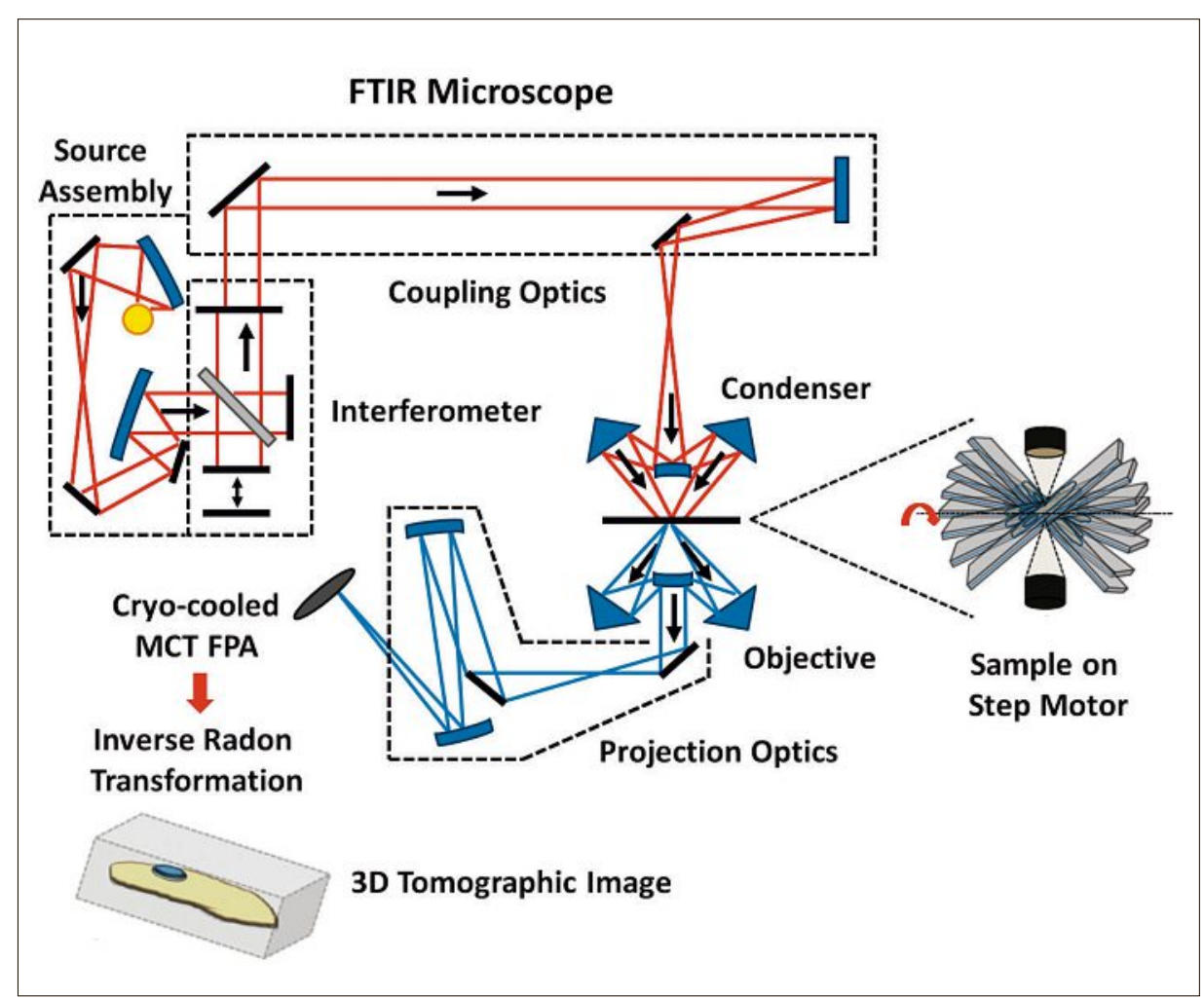

Fig. 1. Schematic representation of an FTIR microscope configured for three-dimensional tomographic chemical imaging. Optical scheme is adapted from ref. [3].

Two basic experimental configurations can be envisioned with an IR microscope: a spectromicroscopic (or microspectroscopic) and an imaging configuration. In a spectromicroscopy measurement, an IR microscope is used to condense light into a single, relatively small spot and to collect a chemical spectrum of the illuminated region. For this measurement the microscope is operated in a confocal geometry and the optical path can be set so that measurements can be performed in either a transmission or a reflection mode. Confocal apertures, located in optical planes conjugated with the samples plane, are used to adjust the size of the incoming and/or outgoing beam and the field of view observed by the detector. By closing the confocal apertures of the objective, a portion of the analyte can be measured with increasing spatial resolution. The performance of confocal IR microscopes is poor when they are operated at high spatial resolution (with apertures closed down to a few $\mu \mathrm{m}$ ). This is due to the important loss of light throughput imposed by the small apertures on the IR beam. Conventional benchtop IR sources are particularly affected in this case because of their limited brightness. As a consequence it is usually difficult to perform IR spectromicroscopy measurements with apertures closed to less than $40 \mu \mathrm{m}$ particularly with complex systems like a cell and with relatively weak absorption bands. In IR microscopy, just as is the case for visible microscopy, the resolution limit is given by the Rayleigh criterion $d=0.61 \lambda / \mathrm{NA}$. For commercial objectives, typical values for the numerical aperture, NA, are 0.4-0.6, which gives a resolution of approximately $\lambda$.

For light in the mid-IR region, diffraction-limited resolution is in the range $2.5-25 \mu \mathrm{m}$. This is of the order of the size of eukaryotic cells, which vary in dimensions from a few to a hundred micrometers. The size of the confocal aperture can be reduced when using synchrotron sources, because of the higher brightness, down to a size allowing diffraction limited resolution and good spectral quality.

With the configuration in Fig. 1, spectromicroscopy measurements can be used to obtain a $2 \mathrm{D}$ distribution of the spectra for each point of the sample by raster scanning. The resulting spectral data can provide a map of specific spectral features, such as the intensity of selected absorption bands, throughout the sample. This procedure is called IR mapping. Nowadays, detectors composed of 2D arrays of elements, called Focal Plane Array (FPA) detectors, allow an IR absorption spectrum to be collected for each pixel position. This results in the simultaneous collection of several thousand spectra covering an extended region of the sample. As with spectromicroscopy mapping, plotting the spatial distribution of specific absorption bands provides an infrared image of the sample. The expression 'IR imaging' is currently applied to the collection of IR images with an FPA detector.

\section{3D FTIR Spectro- Microtomography}

Infrared spectro-tomography measurements can be performed with microscope configurations mentioned in Section 2. The fundamental difference being that while in conventional $2 \mathrm{D}$ IR spectromicroscopy and imaging the sample is static on a suitable support, in a tomography setup the sample is suspended and rotated around the length of the axis arranged perpendicular to the beam path. The rotation can be performed over any range of angles with the number of steps between each rotation determining the number of projection images (PI) to be integrated and thereby the size of the data collection files. Generally speaking, steps of $2^{\circ}$ covering at least $180^{\circ}$ are sufficient to fully sample the image in Fourier space and they will provide a good enough data set for the tomographic reconstruction of the analyte 3D structure. However, the entire Fourier space may have to be probed for very heterogeneous samples. The lateral dimensions perpendicular to the rotation axis of a sample thereby is limited as the entire area of interest should stay in focus during the rotation series.

No commercial sample step motor, or sample holder is currently available to perform infrared spectro-tomography measurements. The specific apparatus and its configuration need to be custom made. Several possibilities can however be envisioned. We used for example a stepper motor with a planetary gearhead whose precise rotary motion was computer controlled (Fig. 2) and a litholoop (Molecular Dimensions) of the type commonly used in X-ray crystallography as sample holder. ${ }^{[4]}$ Others have designed sample holders housed on a goniometer base magnetically mounted onto the head of a threaded socket screw with a permanent magnet glued into the socket hole in combination with a tip-tilt optical mount attached to a piezo-driven rotation stage. ${ }^{[2]}$

In a tomography setup, for each projection image a measurement of the $2 \mathrm{D}$ representation of the sample composition is first obtained by mapping the area or intensity of specific absorption bands over the sample projection surface. Single channel spectra are obtained by performing a Fourier transformation of the interferogram after apodization with a suitable function and phase correction. Data can be collected over the entire spectral range and the area of specific absorptions or complex bands and multiplets can be determined by direct integration of bands above the baseline. The intensity and area of specific bands that are components of complex multiplets can be further extracted by curve fitting 
by e.g. Gaussian/Lorentzian bandshapes to the multiplet. For each projection image this procedure allows the collection of reconstruction data in four dimensions: three spatial dimensions plus a full midIR spectral dimension providing therefore a wealth of information. A 'full-color' spectro-microtomogram, in which specific colors are assigned to specific chemical fingerprints or spectral changes, is finally generated via a suitable algorithm.

For such 3D reconstruction, several algorithms are available such as the algebraic reconstruction technique, ${ }^{[5]}$ the serial iterative reconstruction technique ${ }^{[6]}$ or compressed sensing reconstruction. ${ }^{[7]}$ In general, starting intensity, width, position and number of bands can be chosen so as to provide a first approximation including all the peaks and shoulders in the experimental IR spectrum. Intensity, width and position of all bands, plus the factors of the linear baseline, can then all be floated until convergence and this single spectrum fit is then used as the starting model to automatically fit all the spectra in the pixels of all projections. The resulting intensity values (or the derived band areas) are used for mapping the corresponding bands throughout the projections. In FTIR spectro-tomography the resolution remains a critical factor and the considerations given at the end of Section 2 still apply for the projection images (PI) measurement but the Crowther criterion can be used for a 3D object. In order to determine the resolution $(r)$ that can be achieved for a given number of PI over e.g. $180^{\circ}(N)$ for a sample of size $(s)$, the equation $r=\mathrm{PI} \times$ $s / N$ applies.

\section{Chemical Probes for Cellular 2D IR Imaging}

IR microscopy is extensively used in the study of subtle changes of cells and tissues particularly in health and plant science. Here, however, we focus our discussion on the use of molecular chemical probes that have bridged the gap between live cell IR imaging and inorganic/organometallic chemistry and direct the reader to recent reviews on probe-free applications of the technique. ${ }^{[8]}$ While cyanide, isocyanide and nitrosyl metal complexes are all in principle amiable to IR bio-imaging applications, it is exclusively the carbonyl species that have been considered as FTIR cellular imaging probes. Such complexes are wellknown for their unique infrared signature and show energetically isolated vibrations in a region of the spectrum (typically in the $2150-1750 \mathrm{~cm}^{-1}$ region) where normally no absorptions of organic components are observed. Furthermore, the relatively high extinction coefficient associated with the

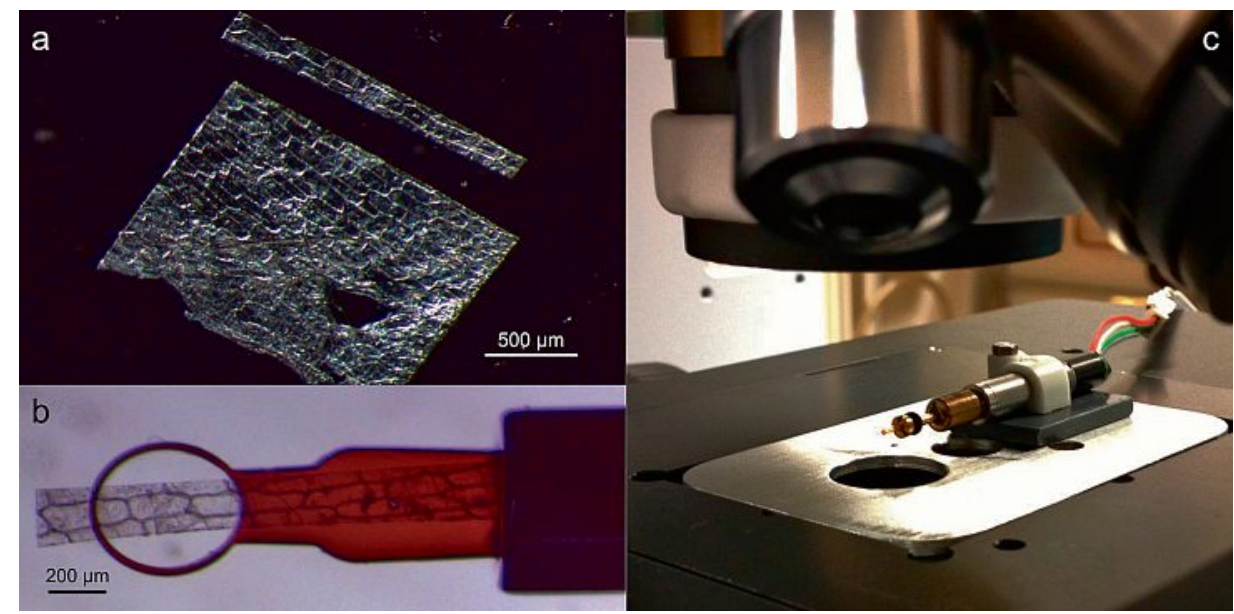

Fig. 2. The experimental setup used by the authors in the measurement of tilt-series transmission images of a single Allium cepa cell: (a) epidermis of Allium cepa from which the single layer of cells was obtained; (b) layer of cells mounted on litholoop sample holder; (c) stepper motor with a planetary gearhead whose precise rotary motion was computer controlled. ${ }^{[4]}$

$\mathrm{M}-\mathrm{C} \equiv \mathrm{O}$ absorption bands allows imaging of the molecules at concentrations of $100 \mu \mathrm{M}$ and lower.

The majority of cellular imaging studies with carbonyl probes have been conducted at synchrotron facilities where the bright source of the radiation allows to focus a greater number of photons onto a given area of the analyte as compared to conventional thermal sources coupled to standard benchtop IR spectrometers. ${ }^{[9]}$ The combination of the synchrotron radiation and IR microscopes has thus enabled the collection of IR maps of cells $(<25 \mu \mathrm{m}$ in size) with subcellular resolution. Nevertheless, the first example of IR bioimaging of an inorganic complex was published by the group of Leong in 2007 by using a Varian 7000 FT-IR spectrometer fitted with a Stingray infrared microscope equipped with a $64 \times 64$ mercury cadmium telluride (MCT) FPA detector. ${ }^{[10]}$ In order to improve sensitivity, and thereby the signal-to-noise ratio, the authors chose to graft osmium-carbonyl clusters (containing up to $10 \mathrm{Os-CO}$ units) to fatty acids and showed that the cellular distribution of the conjugates could be detected at a $32 \mu \mathrm{M}$ concentration. 2D and 3D images of the cellular clusters distribution were then constructed by analysis of the stretching vibrations in the $2010 \mathrm{~cm}^{-1}$ region and were shown to closely correlate with the optical image.

Over the past ten years, group seven metal complexes of $\mathrm{fac}-\left[\mathrm{Mn}(\mathrm{CO})_{3}\right]^{+}$and fac- $\left[\operatorname{Re}(\mathrm{CO})_{3}\right]^{+}$cores have essentially monopolized the IR imaging field. The reasons are manifold. On the one hand, the synthetic procedures to metal species of these cores are now well established and if properly designed the molecules can be directed to specific cellular compartments. ${ }^{[11]}$ On the other hand, a large number of such complexes are the focus of medicinal in- organic studies and are considered as promising candidates in e.g. cancer therapy $^{[12]}$ or as CO-releasing molecules for a wide range of therapeutic applications. ${ }^{[13]}$ The interest in these species is, therefore, dictated not only by their potential value as IR probes, but also by the need to correlate their cellular and tissue distribution with their mode of action.

To this end, in 2012 the group of Policar studied via synchroton radiation FTIR spectromicroscopy the distribution of a cytotoxic rhenium-tricarbonyl tamoxifen analog ${ }^{[14]}$ and a related fluorescent species $^{[15]}$ (probe A in Fig. 3) in MDAMB-231 breast cancer cells. In the hormone-independent cancer cells subcellular levels of the compounds were detected. Single-cell IR mapping showed that the rhenium-tricarbonyl tamoxifen analog matched the location of amide I-bands in an area associated with a cellular bulge that was topographically interpreted as indication of the nucleus. IR spectra recorded inside the bulge revealed IR-signatures of both stretching modes of the fac$\left[\operatorname{Re}(\mathrm{CO})_{3}\right]^{+}$core. These were undetected in other regions of the same cells providing a strong case for the nuclear localization/ imaging of the tamoxifen rhenium-tricarbonyl probe. Conversely, probe A accumulated in the Golgi apparatus with fluorescence imaging confirming the perinuclear localization of the probe. Further results provided evidence for the integrity of the probe in the intracellular environment underlining the reliability of IR imaging.

Similar experiments were performed in 2015 by incubating 3T3 fibroblasts with the anticancer vitamin $\mathrm{B}_{12}$-conjugate B (Fig. 3). ${ }^{[16]}$ IR mapping at diffraction limited spatial resolution revealed the presence of the molecule throughout the cytoplasmic region of the cells but showed a marked concentration in the proximity 


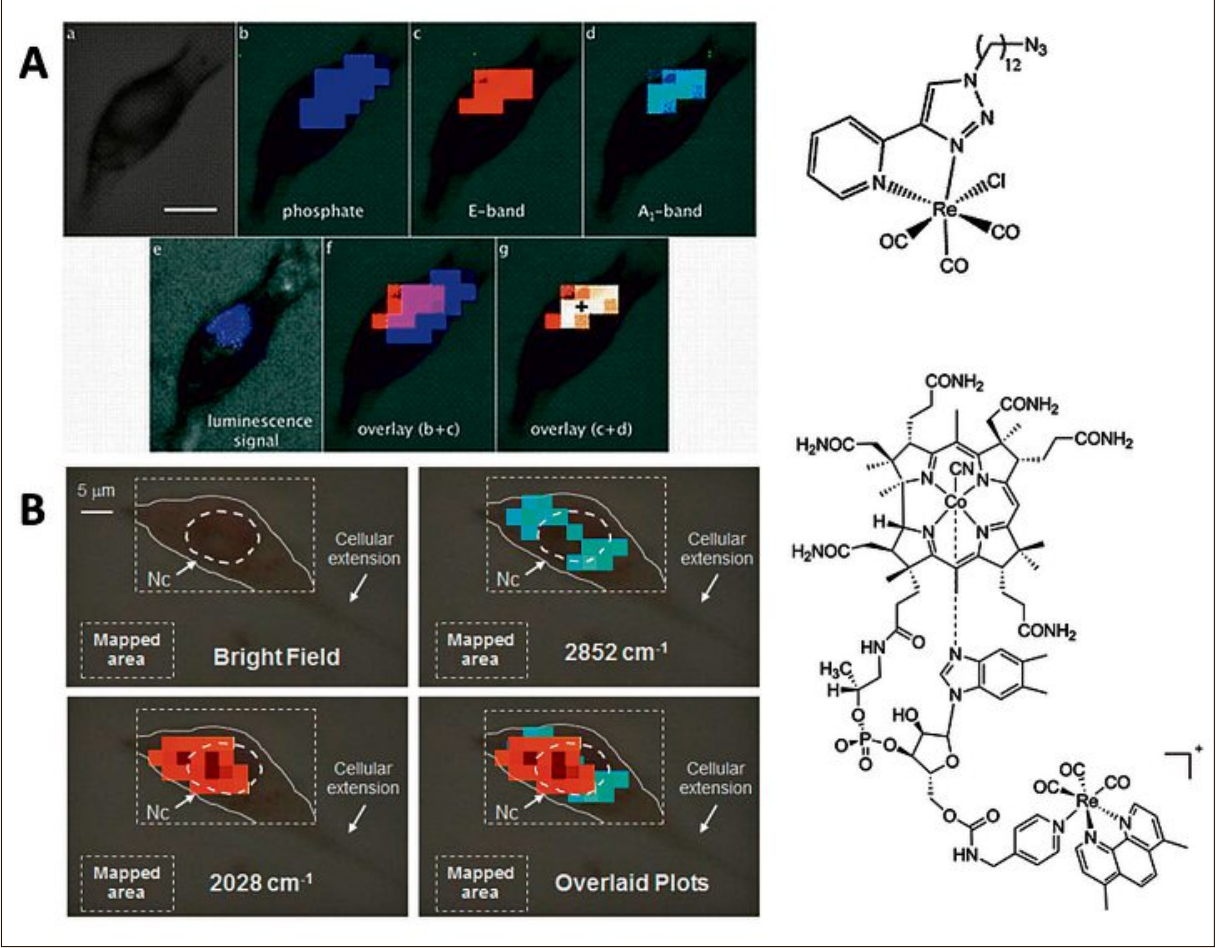

Fig. 3. A. IR imaging of a MDA-MB-231 cell incubated with the Re-based molecular probe to the right of the picture. The different panels show different recorded IR frequencies and the luminescent signal of the probe. Scale bar $10 \mu \mathrm{m}$. Pixel size: $3 \times 3 \mu \mathrm{m}^{2}$. Figure reproduced with permission from ref. [15]. Copyright Royal Society of Chemistry. B. IR imaging of a 3T3 fibroblast incubated with vitamin $\mathrm{B}_{12}$-conjugate to the right of the picture. The different panels show mapping intensities of the lipid absorption at $2852 \mathrm{~cm}^{-1}$ and the $\mathrm{A} 1$ carbonyl stretching vibration of the probe at $2028 \mathrm{~cm}^{-1}$. Scale bar $5 \mu \mathrm{m}$. Pixel size: $3 \times 3 \mu \mathrm{m}^{2}$. Figure adapted with permission from ref. [16] . Copyright Royal Society of Chemistry.

of the nucleus. However, a comparison of the Re-CO stretching frequencies with the absorption at $2852 \mathrm{~cm}^{-1}$ disclosed a substantial nuclear accumulation of the conjugate. The $2852 \mathrm{~cm}^{-1}$ band arises from $\mathrm{CH}_{2}$ stretching modes from long alkyl chains and thus it is a marker for the regions of the cell that are rich in membranes, such as mitochondria, the endoplasmic reticulum and the Golgi. ${ }^{[17]} \mathrm{As}$ is clear from the images collected in these experiments (Fig. 3), lipid concentration is highest in the perinuclear environment, where the endoplasmatic reticulum is usually located, and shows a remarkably different distribution as compared to the signals due to the Re-CO stretching mode. This particular study also highlighted that IR microscopy can be used to discriminate the secondary coordination sphere of the probes. Indeed different cellular environments caused shifts (of $c a .8 \mathrm{~cm}^{-1}$ ) of the probe frequencies depending on their phase of accumulation. The theoretical basis for the interpretation of these shifts is published. ${ }^{[18]}$

A comparable $\mathrm{B}_{12}$-conjugate probe, but containing the $\mathrm{fac}-\left[\mathrm{Mn}(\mathrm{CO})_{3}\right]^{+}$core, in the same cell line, was the subject of a study published in 2013. ${ }^{[19]}$ IR spectromicroscopy was used to characterize the intracellular uptake and reactivity of the photosensitive compound, whose $\mathrm{CO}$ releasing properties could be triggered by illumination with visible light. For the first time it was shown that the technique can be used to follow the real time uptake of chemical probes in live cells over the course of a few hours. Moreover, the intracellular reactivity of the probe, evaluated as a function of the release of the $\mathrm{CO}$ ligands following exposure to visible light, was elucidated. The subcellular distribution of the complex could be described by IR mapping and, taking into consideration the role of cellular topography in the quantitative interpretation of the maps, a perinuclear distribution, in the nucleus and/or in its proximity, appeared to be the most likely interpretation of the images obtained.

\section{Examples of FTIR 3D Spectro-Microtomography}

It is only in the past three years that infrared microscopes have been configured for spectro-tomography measurements and to date the literature counts a total of four contributions in the field. The first experimental implementation of 3D FTIR spectro-microtomography was described by Martin et al. in 2013. [2] As mentioned in the preceding sections, the approach involved the measurement of tilt angle series of $2 \mathrm{D}$ transmission images of the same sample, followed by reconstruction of the tomographic image. The technique was applied to intact and untreated biological or materials samples ranging from $c a$. 30 to $100 \mu \mathrm{m}$. These included the tracheary element of Zinnia elegans (Fig. 4), Populus wood fiber, a human hair and an embryonic body colony of stem cells. All samples were mounted on polyimide microloops housed on a computer-driven rotation stage. Fig. 4 shows the type of reconstructed tomographic images attainable by this methodology. In particular Fig. 4 shows the tracheary element of Zinnia elegans (blue-green central element) within the polyimide microloop structure.

In 2014 and 2016 the group of Hirschmugl, who also coordinated the study mentioned above, published two articles where 3D FTIR spectroscopic imaging measurements were respectively applied to study the phase separation of a poly(3-hydroxybutyrate) (PHB)/poly(L-lactic acid) (PLA) polymer blend film ${ }^{[20]}$ and spectral in situ imaging of an extraterrestrial sample of a $45 \mu \mathrm{m}$ grain of the Murchison meteorite. ${ }^{[21]}$ The first study provided evidence that the technique can discriminate the phase morphology in polymer blends thereby helping to elucidate the factors responsible for the high crystallinity of the thermoplastic PHB polyester, a polymer with great commercial potential. The second study showed how FTIR 3D spectro-microtomography can be used to resolve the spatial relationship between organic and inorganic components in minerals, clarifying their origins and processing. As a nondestructive technique, the method is therefore of great value for the in situ analysis of precious or rare samples, including those of extraterrestrial origin.

Since 2013, we have also been working in parallel and independently to develop FTIR spectro-microtomography as a methodology to address a fundamental problem associated with 2D IR imaging of cells. Conventional cellular 2D IR imaging measurements are restricted in the possibility of associating specific chemical and compositional information to subcellular structures due to the complex topography of the sample. While information in the $z$ direction is being collected with every image, the spectroscopic data associated with a 2D IR imaging experiment can be coupled to the spatial information only in the $x y$ plane and provide a meaningful representation of the chemical composition of the sample only in this plane. Despite the wealth of information in a 2D IR image projection the $z$-dependent information is superimposed, thereby complicating any analysis. Furthermore, unlike other mi- 

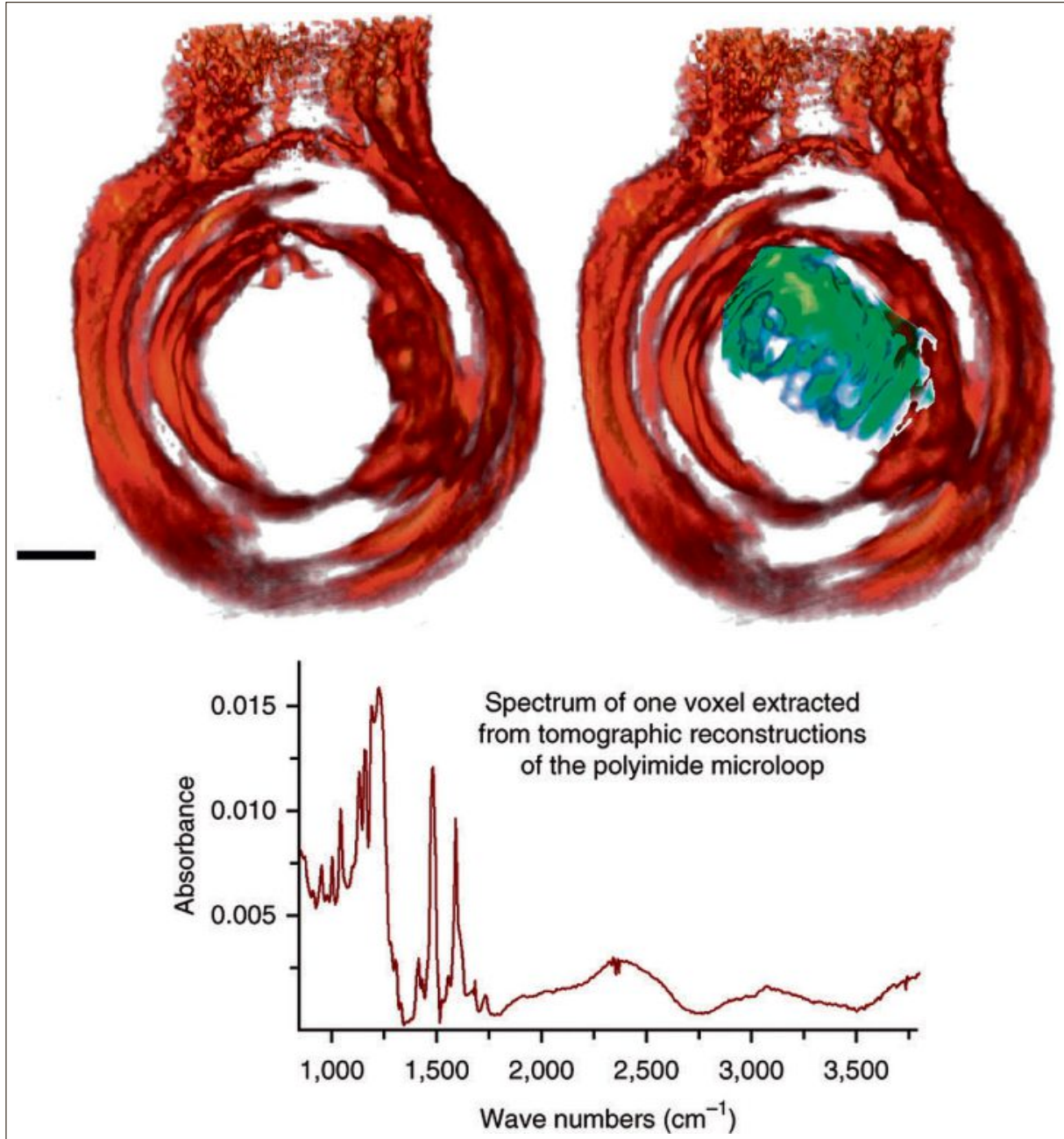

Fig. 4. FTIR spectro-microtomographic imaging of Zinnia elegans tracheary element inside a polyimide microloop. The red color shows the reconstruction of the integrated intensity of polyimide (2356.7-2376.0 $\mathrm{cm}^{-1}$ ) while the blue-green color depicts the reconstruction of the $Z$. elegans element $\left(3317-3340 \mathrm{~cm}^{-1}\right)$. Bottom, spectrum extracted from one $1.1 \times 1.1 \times 1.1 \mathrm{\mu m}^{3}$ voxel showing a polyimide spectrum. Scale bar, $20 \mu \mathrm{m}$. Figure reproduced with permission from ref. [2]. Copyright Nature Publishing Group.

croscopy configurations, like Raman or fluorescent microscopy, where the shorter wavelength of the probing radiation and the shorter depth of focus facilitate 3D image reconstruction by confocal depth profiling, this is not possible with mid-IR radiation, whose depth of focus is frequently greater than cell thickness. The case is particularly relevant if IR imaging is applied to the elucidation of the intracellular localization of drugs.

We have addressed these challenges by developing and applying FTIR spectromicrotomography as a method to collect three-dimensional IR tomographic images of cellular samples and published the first $3 \mathrm{D}$ reconstruction of endogenous and exogenous molecules in a single intact cell. ${ }^{[4]}$ While the basis of the approach had by that time been described by Martin et al., we showed in our study that the chemical imaging capability of mid-IR tomography for subcellular imaging can be fully implemented with a benchtop microscope and a conventional light source. This is a significant step forward because the need for synchrotron light considerably restricts the applicability of the method. In our study, we reconstructed the $3 \mathrm{D}$ distribution of molecular components in a single Allium cepa cell by measuring tilt-series of their transmission images (Fig. 5). The tomographic images reconstructed from selected absorption bands clearly rendered the spatial distribution of subcellular structures, including the cell wall, the nucleus, and large vacuoles. These objects were as little as $8.5 \mu \mathrm{m}$ apart, suggesting that the resolution of our technique is close to diffraction-limited values.

In the same study, we further tested the use of IR tomography for the subcellular localization of an exogenous compound by mapping the 3D distribution of the fac$\left[\mathrm{Re}(\mathrm{CO})_{3}\left(\mathrm{H}_{2} \mathrm{O}\right)_{3}\right]^{+}$ion. The tomographic images of the corresponding stretching bands of the complex indicated preferential accumulation of the molecule within the cell wall and the nucleus (Fig. 5). We noticed that the accumulation of the ion within the lipid body was negligible, a feature that would be difficult to appreciate in 2D images. As mentioned in Section 4, previous 2D IR imaging and mapping experiments addressing the cellular distribution of compounds related to the $f a c-\left[\operatorname{Re}(\mathrm{CO})_{3}\left(\mathrm{H}_{2} \mathrm{O}\right)_{3}\right]^{+}$ion were complicated by difficulties in interpreting IR maps in the proximity of the nucleus because of interfering cellular topography (Fig. 3). In contrast, 3D images obtained by FTIR spectro-microtomography are unambiguous and the comparison of the relative ratios of several absorption bands of the same compound even allowed for conclusions on the coordination of the molecule within different compartments of the cell.

\section{Conclusions}

Mid-IR absorption spectroscopy in its spectromicroscopy configuration is widely employed in many fields of science. As a nondestructive, label-free technique it is useful for both compositional analysis and the characterization of molecular properties of a wide variety of samples, including rare or precious materials. The recent implementation in spectro-tomography measurements has significantly enhanced the probing potentials of IR-imaging now allowing for the reconstruction of complex 3D structures. The technique is still in its infancy and its full potential is yet to be explored but we believe the method is now poised to make a significant contribution in a wide variety of scientific, industrial, materials, energy and medical applications. Advances in FPA detector technology and computational tomography will certainly improve in the future the probing capacities of FTIR spectro-microtomography.

\section{Acknowledgements}

The authors are indebted to Dr. Luca Quaroni (Jiagiellonian University, Faculty of Chemistry, Kraków) for his invaluable help in the preparation of this contribution.

Received: December 19, 2016

[1] V. Bobroff, H. H. Chen, S. Javerzat, C. Petibois, J. Biophotonics 2016, 129, 1.

[2] M. C. Martin, C. Dabat-Blondeau, M. Unger, J. Sedlmair, D. Y. Parkinson, H. A. Bechtel, B. Illman, J. M. Castro, M. Keiluweit, D. Buschke, B. Ogle, M. J. Nasse, C. J. Hirschmugl, Nat. Methods 2013, 10, 861.

[3] J. A. Rowlette, M. J. Weida, B. Bird, D. F. Arnone, M. Barre, T. Day, BioOptics 2014, 7, 34.

[4] L. Quaroni, M. Obst, M. Nowak, F. Zobi, Angew. Chem. Int. Ed. 2015, 54, 318.

[5] G. T. Herman, A. Lent, S. W. Rowland, J. Theor. Biol. 1973, 42, 1 .

[6] P. Gilbert, J. Theor. Biol. 1972, 36, 105. 


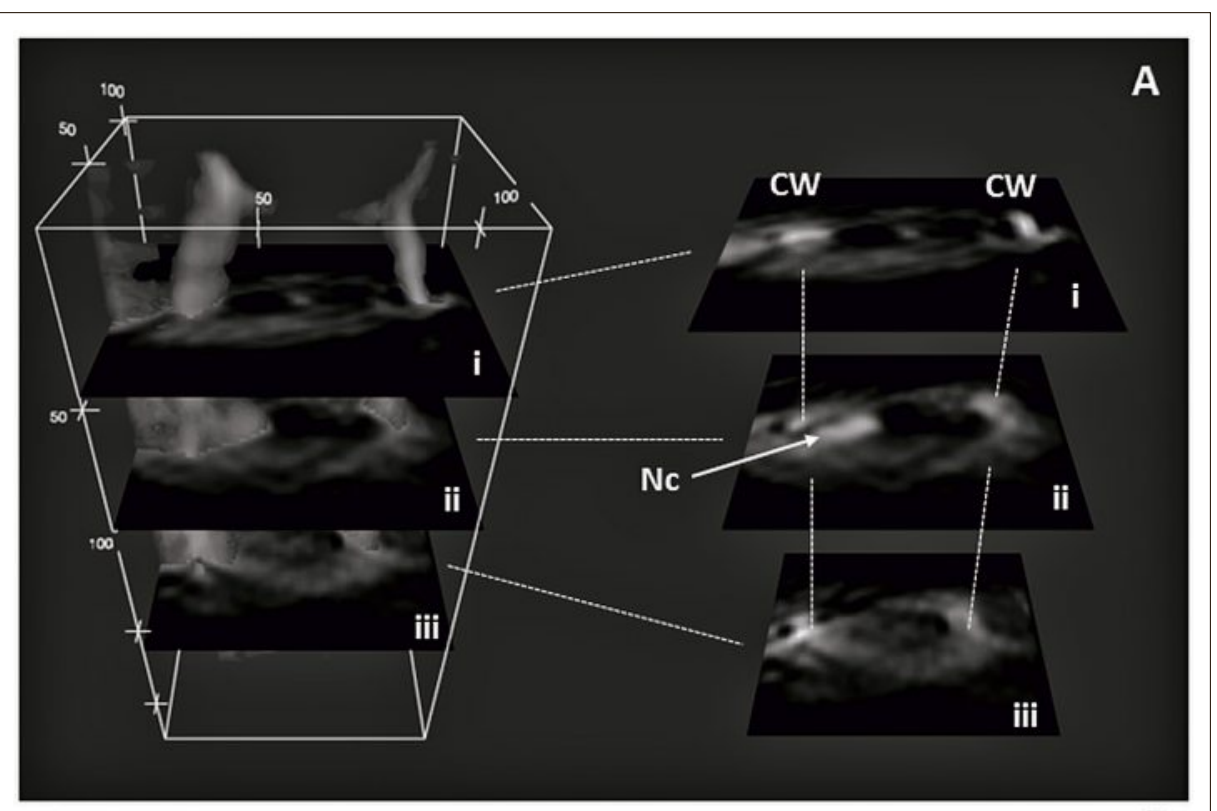

B

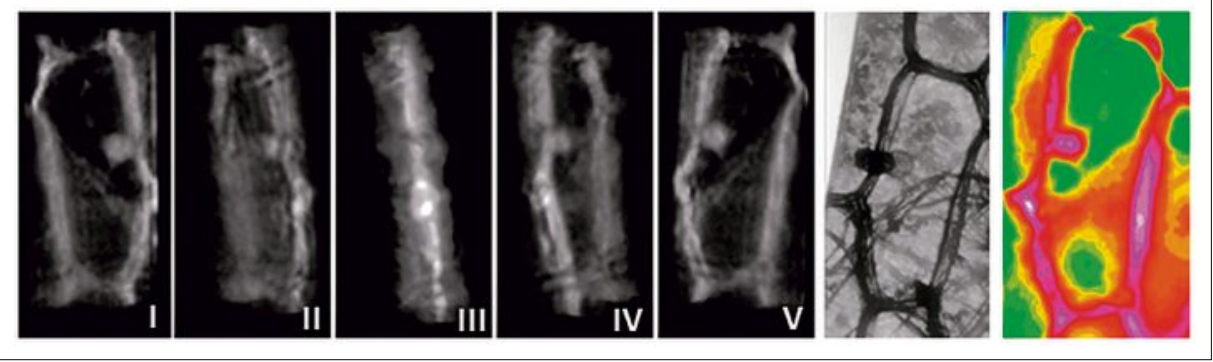

Fig. 5. 3D computed tomography reconstruction of the cellular distribution of the fac$\left[\operatorname{Re}(\mathrm{CO})_{3}\left(\mathrm{H}_{2} \mathrm{O}\right)_{3}\right]^{+}$probe in a single Allium cepa cell. A. Reconstructed volume rendering with digital slicing at selected locations. B. Volume rendering visualized as a function of rotation about the vertical axis. The rotation series is accompanied by the optical image of the cell and a single 2D projection image. Figure adapted with permission from ref. [4]. Copyright WILEY-VCH Verlag GmbH \& Co. KGaA, Weinheim.

[7] Z. Saghi, D. J. Holland, R. Leary, A. Falqui, G. Bertoni, A. J. Sederman, L. F. Gladden, P. A. Midgley, Nano Lett. 2011, 11, 4666.

[8] a) M. Diem, M. Miljkovic, B. Bird, T. Chernenko, J. Schubert, E. Marcsisin, A. Mazur, E. Kingston, E. Zuser, K. Papamarkakis, N. Laver, Spectrosc.-Int. J. 2012, 27, 463; b) M. J. Baker, J. Trevisan, P. Bassan, R. Bhargava, H. J. Butler, K. M. Dorling, P. R. Fielden, S. W. Fogarty, N. J. Fullwood, K. A. Heys, C. Hughes, P. Lasch, P. L. Martin-Hirsch, B. Obinaju, G. D Sockalingum, J. Sule-Suso, R. J. Strong, M. J. Walsh, B. R. Wood, P. Gardner, F. L. Martin Nat. Protoc. 2014, 9, 1771; c) D. C. Fernandez, R. Bhargava, S. M. Hewitt, I. W. Levin, Nature Biotechnol. 2005, 23, 469; d) G. Bellisola, C. Sorio, Am. J. Cancer Res. 2012, 2, 1; e) S. G. Kazarian, K. L. A. Chan, Analyst 2013, 138 , 1940; f) E. C. Mattson, E. Aboualizadeh, M. E. Barabas, C. L. Stucky, C. J. Hirschmugl, Int. J. Mol. Sci. 2013, 14, 22753; g) J. Doherty, G. Cinque, P. Gardner, Appl. Spectrosc. Rev. 2016 45, 1935.

[9] S. Clede, C. Policar, Chem. Eur. J. 2015, 21, 942.

[10] K. V. Kong, W. Chew, L. H. K. Lim, W. Y. Fan, W. K. Leong, Bioconjugate Chem. 2007, 18, 1370.

[11] a) F. L. Thorp-Greenwood, R. G. Balasingham, M. P. Coogan, J. Organomet. Chem. 2012, 714 12; b) R. G. Balasingham, M. P. Coogan, F. L. Thorp-Greenwood, Dalton Trans. 2011, 40,
11663; c) V. Fernandez-Moreira, F. L. ThorpGreenwood, A. J. Amoroso, J. Cable, J. B. Court, V. Gray, A. J. Hayes, R. L. Jenkins, B. M. Kariuki, D. Lloyd, C. O. Millet, C. F. Williams, M. P. Coogan, Org. Biomol. Chem. 2010, 8, 3888; d) A. J. Amoroso, R. J. Arthur, M. P. Coogan, J. B. Court, V. Fernandez-Moreira, A. J. Hayes, D. Lloyd, C. Millet, S. J. A. Pope, New J. Chem. 2008, 32, 1097; e) A. J. Amoroso, M. P. Coogan, J. E. Dunne, V. Fernandez-Moreira, J. B. Hess, A. J. Hayes, D. Lloyd, C. Millet, S. J. A. Pope, C. Williams, Chem. Commun. 2007, 3066.

[12] a) J. Y. Zhang, J. J. Vittal, W. Henderson, J. R. Wheaton, I. H. Hall, T. S. A. Hor, Y. K. Yan, J. Organomet. Chem. 2002, 650, 123; b) M. D. Bartholoma, A. R. Vortherms, S. Hillier, B. Ploier, J. Joyal, J. Babich, R. P. Doyle, J. Zubieta, Chemmedchem 2010, 5, 1513; c) D. Can, H. W. P. N'Dongo, B. Spingler, P. Schmutz, P. Raposinho, I. Santos, R. Alberto, Chem. Biodiversity 2012, 9, 1849; d) A. W. T. Choi, M. W. Louie, S. P. Y. Li, H. W. Liu, B. T. N. Chan, T. C. Y. Lam, A. C. C. Lin, S. H. Cheng, K. K. W. Lo, Inorg. Chem. 2012, 51, 13289; e) J. M. Ho, W. Y. Lee, K. J. T. Koh, P. P. F. Lee, Y. K. Yan, J. Inorg. Biochem. 2013, 119, 10; f) A. Leonidova, G. Gasser, ACS Chem. Biol. 2014, 9, 2180; g) A. Leonidova, V. Pierroz, R. Rubbiani, J. Heier, S. Ferrari, G. Gasser, Dalton Trans. 2014, 43, 4287.

[13] a) F. Zobi, Future Med. Chem. 2013, 5, 175; b)
F. Zobi, O. Blacque, R. A. Jacobs, M. C. Schaub, A. Y. Bogdanova, Dalton Trans. 2012, 41, 370; c) H. B. Suliman, F. Zobi, C. A. Piantadosi, Antioxid. Redox Signal. 2016, 24, 345.

[14] S. Clede, F. Lambert, C. Sandt, Z. Gueroui, N. Delsuc, P. Dumas, A. Vessieres, C. Policar, Biotechnol. Adv. 2013, 31, 393.

[15] S. Clede, F. Lambert, C. Sandt, Z. Gueroui, M. Refregiers, M. A. Plamont, P. Dumas, A. Vessieres, C. Policar, Chem. Commun. 2012 48,7729 .

[16] G. Santoro, T. Zlateva, A. Ruggi, L. Quaroni, F. Zobi, Dalton Trans. 2015, 44, 6999.

[17] G. van Meer, D. R. Voelker, G. W. Feigenson, Nat. Rev. Mol. Cell Bio. 2008, 9, 112.

[18] a) F. Zobi, Inorg. Chem. 2009, 48, 10845; b) F. Zobi, Inorg. Chem. 2010, 49, 10370.

[19] F. Zobi, L. Quaroni, G. Santoro, T. Zlateva, O. Blacque, B. Sarafimov, M. C. Schaub, A. Y. Bogdanova, J. Med. Chem. 2013, 56, 6719.

[20] M. Unger, J. Sedlmair, H. W. Siesler, C. Hirschmugl, Vib. Spectrosc. 2014, 75, 169.

[21] M. Yesiltas, J. Sedlmair, R. E. Peale, C. Hirschmugl, Appl. Spectrosc. 2016, DOI: $10.1177 / 0003702816671072$ 